\title{
HEIGHT AND WIDTH OF SUPERATOMIC BOOLEAN ALGEBRAS
}

\author{
JUDY ROITMAN ${ }^{1}$
}

\begin{abstract}
Cantor-Bendixson height and width of superatomic Boolean algebras is investigated and it is shown that (1) you don't need a Canadian tree to construct an $\omega_{1}$-thin-thick superatomic Boolean algebra;

(2) $\mathbf{c}$ can be very large and for all $\kappa<\mathbf{c}$ and all uncountable $\lambda, \kappa<\mathbf{c}$, there are no $\kappa$-thin-very tall, $\lambda$-thin-tall, $\kappa$-very thin-thick, or $\lambda$-thin-thick superatomic Boolean algebras on $\kappa$.
\end{abstract}

0. Introduction. A superatomic Boolean algebra (henceforth abbreviated $\mathrm{sBa}$ ) is a Boolean algebra in which every subalgebra (equivalently, every quotient algebra) is atomic. The class of sBa's is exactly the class of Boolean algebras whose Stone spaces are compact scattered; hence, the results of this paper have direct translations into the theory of compact scattered spaces.

Let $X$ be a Boolean algebra. For each ordinal $\alpha$ we define the $\alpha$ th Cantor-Bendixson ideal $J_{\alpha}$ on $X$ as follows: $J_{0} \neq \varnothing$; given $J_{\alpha}$, let $A t^{\alpha}(X)=\left\{x: x / J_{\alpha}\right.$ is an atom of $\left.X / J_{\alpha}\right\}$ and let $J_{\alpha+1}$ be the ideal generated by $J_{\alpha} \cup A t^{\alpha}(X)$; given $J_{\beta}$ for all $\beta<\alpha, \alpha$ a limit, let $J_{\alpha}=\cup_{\beta<\alpha} J_{\beta} . X$ is an sBa iff, for some $\alpha, X=J_{\alpha}$.

Suppose $X$ is an $\mathrm{sBa}$. For each $x \in X$ we define $\operatorname{rank}(x)$ to be the least $\alpha$ with $x \in J_{\alpha+1}-J_{\alpha}$. The Cantor-Bendixson height of $X$, ht $(X)$, is the least $\alpha$ such that $X=J_{\alpha}$; note that $\mathrm{ht}(X)$ is always a successor ordinal. For each ordinal $\alpha$, let $\operatorname{wd}_{\alpha}(X)=\left|J_{\alpha+1} / J_{\alpha}\right|$ (i.e. the number of atoms in $X / J_{\alpha}$ ), and define the CantorBendixson width of $X, \operatorname{wd}(X)$, to be the supremum of all $\mathrm{wd}_{\alpha}(X)$.

An $\mathrm{sBa} X$ is

(a) $\kappa$-thin iff $\operatorname{wd}(X)=\kappa$. (Note: thin $=\omega$-thin.)

(b) $\kappa$-thin-thick iff $\operatorname{wd}_{\alpha}(X)=\kappa$ for $\alpha<\kappa$ and $\operatorname{wd}_{\kappa}(X)=\kappa^{+}$. (Note: thin-thick $=$ $\omega_{1}$-thin-thick $\neq$ Just's thin-thick.)

(c) $\kappa$-very-thin-thick iff $w_{\alpha}(X)<\kappa$ for $\alpha<\kappa$ and $\operatorname{wd}_{\kappa}(X)=\kappa^{+}$. (Note: very-thinthick $=\omega_{1}$-very-thin-thick $=$ Just's thin-thick.)

(d) $\kappa$-thin-very-thick iff $w_{\alpha}(X)=\kappa$ for $\alpha<\kappa$ and $\operatorname{wd}_{\kappa}(X)=\kappa^{++}$.

(e) $\kappa$-thin-tall iff $X$ is $\kappa$-thin and $\operatorname{ht}(X)=\kappa^{+}$. (Note: thin-tall $=\omega$-thin tall.)

(f) $\kappa$-thin-very tall iff $X$ is $\kappa$-thin and $h t(X)=\kappa^{++}$. (Note: thin very tall $=\omega$-thinvery tall.)

The existence of thin-tall sBa's was shown by Rajagapolan and, independently, by Juhász and Weiss. Just showed the consistency of no thin-very tall sBa's and no very

Received by the editors January 24, 1984.

1980 Mathematics Subject Classification. Primary 06E99, 03 E35.

${ }^{1}$ This research partially supported by NSF grant \# MCS-8102599.

(C)1985 American Mathematical Society $0002-9939 / 85 \$ 1.00+\$ .25$ per page 
thin-thick sBa's. Weese noticed that the existence of a Canadian tree implies the existence of a thin-thick $\mathrm{sBa}$ and wondered if the implication reversed. This paper shows that

(1) Modulo the consistency of an inaccessible cardinal (an inescapable modification) Weese's implication does not reverse. The construction generalizes to, e.g., thin-very thick sBa's, but it is not yet known if the combinatorial principle needed there is consistent with no Canadian trees.

(2) Just's results generalize wildly; in particular his models have no thin-thick or $\omega_{1}$-thin-tall sBa's with countably many atoms. (For an exact statement of the generalizaiton see §3.)

After this paper was written, Baumgartner showed Cons (there is no thin-thick $\mathrm{sBA}$ ), and I have show Cons (there is a very thin-thick sBA).

Some set theory conventions: $|A|$ is the cardinality of $A$; Greek letters denote ordinals (hence sometimes cardinals); $\kappa, \lambda$ always denote cardinals; MA is Martin's axiom; $\mathrm{CH}$ is the continuum hypothesis; $\mathrm{c}$ is the size of the continuum; for a statement $P$, Cons $(P)$ is the statement " $P$ is consistent."

1. Thin-thick sBa's. A Canadian tree is a tree of height $\omega_{1}$, size $\omega_{1}$, and at least $\omega_{2}$ uncountable branches. Weese's thin-thick sBa from a Canadian tree is the Boolean algebra generated by the set of uncountable branches. We use a different combinatorial structure to build thin-thick sBa's.

Definition 1. Two sets are almost disjoint iff their intersection is at most finite. (Note: this coincides with the usual definition only when the sets are countable.)

THEOREM 2. Suppose there is a pairwise almost disjoint family of size $\kappa \geqslant \omega_{2}$ whose members are uncountable subsets of $\omega_{1}$. Then there is a thin-thick $s B a X$ with $\operatorname{wd}_{\omega_{1}}(X)=\kappa$.

Proof. Let $\mathscr{A}=\left\{A_{\gamma}: \gamma<\kappa\right\}$ be the family of the hypothesis. We may assume $\mathrm{U}_{\gamma<\kappa} A_{\gamma}=\omega_{1}$. The idea is to have $\omega_{1}$ many sBa's of Cantor-Bendixson width $\omega_{1}$ and arbitrarily large countable height, take their direct limit, and, at the $\omega_{1}$-level, use the $A_{\gamma}$ 's to glue chunks together. The pairwise almost disjointedness of $\mathscr{A}$ will then ensure that pairwise intersections of elements on the $\omega_{1}$ st level will have rank less than $\omega_{1}$.

So let $\left\{B_{\beta}: \beta<\omega_{1}\right\}$ partition $\omega_{1}$ into uncountable sets and for each $\beta<\omega_{1}$ let $X^{\beta}$ be an $\mathrm{sBa}$ of height $\beta+1$ with $\operatorname{wd}\left(X^{\beta}\right)=\omega_{1}$ and $\cup X^{\beta}=B_{\beta}$. Write the $\alpha$ th Cantor-Bendixson ideal of $X^{\beta}$ as $J_{\alpha}^{\beta}$.

Our thin-thick sBa $X$ is the Boolean algebra generated by $\bigcup\left\{J_{\alpha}^{\beta}: \alpha, \beta<\omega_{1}\right\} \cup\left\{A_{\alpha}^{*}\right.$ : $\alpha<\kappa\}$ where $A_{\alpha}^{*}=\bigcup\left\{B_{\beta}: \beta \in A_{\alpha}\right\}$. We must show that $X$ is superatomic, thin-thick, and that $\mathrm{wd}_{\omega_{1}}(X)=\kappa$.

Let $I_{\alpha}$ be the $\alpha$ th Cantor-Bendixson ideal of $X$. An easy inductive proof shows that for $\alpha \leqslant \omega_{1}, \beta<\omega_{1}, I_{\alpha} \supset J_{\alpha}^{\beta}$. Since for $\alpha<\kappa, \beta<\omega_{1}, \operatorname{rank}\left(A_{\alpha}^{*}\right)>\beta$ (because for cofinally many $\beta A_{\alpha}^{*}$ contains an element of $I_{\beta}$ ), no $A_{\alpha}^{*} \in I_{\omega_{1}}$. Since each $A_{\alpha}^{*} \cap A_{\alpha^{\prime}}^{*}$, is a finite union of $B_{\beta}$ 's for $\alpha \neq \alpha^{\prime}$, each $\operatorname{rank}\left(A_{\alpha}^{*}\right)=\omega_{1}+1$ and they are distinct $\bmod I_{\omega_{1}}$. Hence, for $\alpha \leqslant \omega_{1}, I_{\alpha}=\bigcup_{\beta<\omega_{1}} J_{\alpha}^{\beta}$ and $w_{\omega_{1}}(X)=\kappa$. Every element 
of $X$ is either in $I_{\omega_{1}+1}$ or is the complement of an element in $I_{\omega_{1}+1}$ so $X$ is superatomic and we are done.

When does the pairwise almost disjoint family of the hypothesis exist? Not always. For example, if $\mathrm{CH}$ holds, then for any family of size $\omega_{2}$ whose members are infinite subsets of $\omega_{1}$, there is a subfamily of size $\omega_{2}$ and a fixed infinite set contained in every member of the subfamily; that $\mathrm{CH}$ is not necessary for this is a result due to Baumgartner. Baumgartner was also the first to construct such a family, via forcing. We will construct our family by invoking a lemma of Wage that holds under Martin's axiom and then forcing MA over appropriate models of set theory, which give a slightly stronger theorem.

Lemma 3. (Wage) Assume $M A+\mathbf{c}>\kappa$. Then if $\mathscr{A}$ is a family of size $\kappa$ whose elements are countable pairwise almost disjoint subsets of $\omega_{1}$, there is an uncountable $B \subset \cup \mathscr{A}$ with $B \cap A$ finite for all $A \in \mathscr{A}$.

LEMmA 4. Assume $M A+\mathbf{c} \geqslant \kappa$ and suppose there is $\left\{F_{\alpha}: \alpha<\kappa\right\}$, a family of uncountable subsets of $\omega_{1}$ with each pairwise intersection countable. Then there is $a$ pairwise almost disjoint family of size $\kappa$ whose elements are uncountable subsets of $\omega_{1}$.

Proof. We can construct the desired pairwise disjoint family $\left\{E_{\alpha}: \alpha<\kappa\right\}$ as follows: $E_{0}=F_{0}$. If $\left\{E_{\beta}: \beta<\alpha\right\}$ has already been constructed where each $E_{\beta} \subset F_{\beta}$, let $C=F_{\alpha} \cap \cup_{\beta<\alpha} E_{\beta}$. If $F_{\alpha}-C$ is uncountable, let $E_{\alpha}=F_{\alpha}-C$. Otherwise, apply Wage's lemma to $\left\{E_{\beta} \cap F_{\alpha}: \beta<\alpha\right\}$ to construct the desired $E_{\alpha} \subset C \subset F_{\alpha}$.

Lemma 5. Assume $M A+\neg C H$. Then there is a thin-thick $s B a$.

Proof. The family $\left\{F_{\alpha}: \alpha<\omega_{2}\right\}$ of Lemma 4 can be constructed in ZFC using a standard diagonal construction.

Lemma 6. For any cardinal $\kappa \geqslant \mathrm{c}^{+}$Cons (there is a thin-thick $s B a X$ with $\left.\operatorname{wd}_{\omega_{1}}(X)=\kappa\right)$.

Proof. Use standard countably closed conditions to force the existence of $\left\{F_{\alpha}\right.$ : $\alpha<\kappa\}$ as in Lemma 4. (Note: if $\mathrm{CH}$ fails in the ground model, $\mathrm{c}$ gets collapsed to $\omega_{1}$, but that is okay.) Now force $M A+c \geqslant \kappa$ to hold.

Which of the preceding constructions can be put together with the nonexistence of a Canadian tree? At this stage of knowledge, only Lemma 5. The easiest way is to use the proper forcing axiom, PFA, which implies both $M A+\neg C H$ and there are no Canadian trees. However, PFA's consistency is known only from the consistency of a supercompact cardinal. That is stronger than necessary: Baumgartner and Todorcević independently showed that the consistency of an inaccessible cardinal suffices to prove the consistency of $\mathrm{MA}+\neg \mathrm{CH}$ and that there are no Canadian trees. That the inaccessible cardinal is necessary follows from Mitchell's result that the consistency of no Canadian trees is in fact equivalent to the consistency of an inaccessible cardinal. So we have

TheOREM 7. Cons (there is an inaccessible cardinal) implies Cons (there is a thin-thick $s B a$ and there are no Canadian trees ). 
It is not known whether Lemma 6 is a superfluous construction; i.e., whenever Lemma 6 can be invoked there may be a Canadian tree around which will do the job just as well.

2. More preliminaries. Before stating and proving generalizations of Just's results, we need some ad hoc forcing notation. For any set $A$ and any cardinal $\lambda, \mathbf{P}_{A}(\lambda)$ is the set of partial functions from $A$ into 2 whose domains have size less than $\lambda$, ordered under reverse inclusion. E.g. $\mathbf{P}_{\kappa}(\omega)$ is the usual ordering adding $\kappa$ Cohen reals, for $\kappa$ an infinite cardinal. If $\lambda$ is fixed we will just write $\mathbf{P}_{A}$ instead of $\mathbf{P}_{A}(\lambda)$.

Now for some forcing notation and facts.

$M$ will always denote a countable transitive model of set theory. If $\mathbf{P}=\mathbf{P}_{A} \cap M$, $G$ is P-generic over $M$, and $B \subset A$, then we let $M_{B}=M\left[G \cap \mathbf{P}_{B}\right] \subset M[G]$.

Terms are denoted by ${ }^{\circ}$, i.e., $\stackrel{\circ}{x}$ is a term in the forcing language. We will say, e.g. "let $x \in M[G]$ and let $\stackrel{x}{x}$ be a term for $x \ldots$... For $P$ a statement, we abbreviate " $P$ holds in $M$ (resp. $M[G])$ " by “ $M$ (resp. $M[G]) \vDash P$."

Assume $\lambda$ is regular. Recall that $\mathbf{P}_{A}(\lambda) \cap M$ adds no new subsets of $M$ of size less than $\lambda$, and that if, in addition, $\left(\lambda^{+}\right)^{<\lambda}=\lambda^{+}$then $\mathbf{P}_{A}(\lambda) \cap M$ collapses no cardinals of $M$.

If $\mathbf{P}=\mathbf{P}_{A}(\lambda)$ and $\dot{x}$ is a $\mathbf{P}$-term we define $\operatorname{supp}(\dot{x})=\bigcup\{$ dom $p$ : there is some $\langle p, \tau\rangle \in \stackrel{\circ}{x}\}$.

If $\phi$ is an order-preserving permutation of a partial order $\mathbf{P}$, then it induces a permutation of terms, also called $\phi$, so that if for some formula $P \mathbb{1} \Vdash P\left(\dot{x}_{1} \ldots \dot{x}_{n}\right)$ then $1 \Vdash P\left(\phi\left(\dot{x}_{1}\right) \cdots \phi\left(\dot{x}_{n}\right)\right)$. In the particular case $\mathbf{P}=\mathbf{P}_{A}(\lambda) \cap M$ for some $M$, any permutation $\phi$ of $A$ gives rise to an order-preserving permutation of $\mathbf{P}$, also called $\phi$, and hence to a truth-perserving permutation of P-terms. In particular, if $\mathbf{1} \vDash \stackrel{\circ}{x} \subset A$, then for all $a \in A$ and $p \in \mathbf{P}, p \Vdash a \in \dot{x}$ iff $\phi(p) \Vdash \phi(a) \in \phi(\dot{x})$, where the letter $\phi$ denotes, respectively, the permutation of $\mathbf{P}$, of $A$, and of P-terms. For example, $\phi(\stackrel{\circ}{x})=\stackrel{\circ}{x}$ if $1 \Vdash \stackrel{\circ}{x} \subset A$ and

$$
\phi \mid[\operatorname{supp}(\stackrel{\circ}{x}) \cup\{a \in A: \exists p(p \Vdash a \in \stackrel{\circ}{x})\}]
$$

is the identity, where the second $\phi$ is just the original map on $A$.

3. Generalizing Just. We want to show the consistency of: for many $\kappa$ there are no $\kappa$-thin-very tall and no $\kappa$-very thin thick sBa's. In fact we will do better showing the consistency of: for many $\kappa$ there are no $\kappa$-thin-tall sBa's with fewer than $\kappa$ atoms, and no $\kappa$-thin-thick sBa's with fewer than $\kappa$ atoms.

TheOREM 8. Suppose $M \vDash(G C H+\omega \leqslant \lambda<\kappa$ and $\lambda$ is regular $)$; suppose $\mathbf{P}=$ $\mathbf{P}_{A}(\lambda) \cap M$ where $M \vDash|A| \geqslant \kappa^{+}$. Then if $G$ is $\mathbf{P}$-generic over $M$, the following hold in $M[G]$ :

(a) there is no $\kappa$-thin-thick $s B a$ with fewer than $\kappa$-atoms

(b) there is no $\kappa$-thin-tall sBa with fewer than $\kappa$-atoms.

NotEs. 1. GCH is not really necessary, but it makes things more readable. $\left(\lambda^{+}\right)^{<\lambda}=\lambda^{+}$and $2^{<\kappa}=\kappa$ suffice.

2. If, e.g., $\lambda=\omega$, and $|A|$ is a limit cardinal of uncountable cofinality, then the results promised in the abstract and in $\S 0$ appear, since $M[G] \vDash \mathrm{c}=|A|$; hence, 
$M[G] \vDash$ for all uncountable $\kappa<\mathrm{c}$ there are no $\kappa$-thin-thick or $\kappa$-thin-tall sBa's with fewer than $\kappa$ atoms.

3. The proofs of the separate parts of Theorem 8 are close enough to Just's proofs (although in a different mathematical language) to make me uncomfortable claiming them entirely as my own, yet (especially in (b)) far enough away, and the statements so different, as to make me uncomfortable calling them otherwise. The reader is welcome to judge for him/herself; the important matter is communicating the mathematics.

Proof of 8(a). Suppose $X \in M[G]$ is a $\kappa$-thin-thick sBa with fewer than $\kappa$ many atoms. We may assume $\bigcup X=\delta$ for some $\delta<\kappa$. Working for a moment in $M[G]$, $X=\bigcup_{\alpha<\rho} J_{\alpha}$ for some $\rho \geqslant \kappa+1$, where $J_{\alpha}$ is the $\alpha$ th Cantor-Bendixson ideal, each $\left|J_{\alpha}\right| \leqslant \kappa$ for $\alpha<\kappa$, and $\mathrm{wd}_{\kappa}(X)=\kappa^{+}$.

Now we move the proof back to $M$. In $M$ there are terms $\left\{\dot{x}_{\alpha}: \alpha<\kappa^{+}\right\}$where $1 \Vdash\left\{\stackrel{\circ}{x}: \alpha<\kappa^{+}\right\}$generates $A t^{\kappa}(\stackrel{\circ}{x})$. Since $\mathbf{P}$ has the $\lambda^{+}$-chain condition and $\kappa^{+}>\lambda^{+}$, if $\alpha<\beta<\kappa^{+}$then there is $\gamma_{\alpha, \beta}<\kappa$ so $1 \Vdash \dot{x}_{\alpha} \cap \dot{x}_{\beta} \subset \dot{J}_{\gamma_{\alpha, \beta}}$. If we can find in $M[G]$ a fixed $\gamma$ and a set $B$ of size $\kappa^{+}$so that for any distinct $\alpha, \beta \in B, \gamma_{\alpha, \beta}=\gamma$, we will be done, since then $\mathbf{1} \vDash \operatorname{wd}_{\gamma}(\stackrel{\circ}{X}) \geqslant \kappa^{+}$, which is a contradiction.

For each $\alpha<\kappa^{+}$let $S_{\alpha}=\operatorname{supp} \dot{x}_{\alpha}$. Since $\mathbf{P}$ has the $\lambda^{+}$-chain condition and $\mathbf{1} \vDash \stackrel{\circ}{x}_{\alpha} \subset \delta$, we may assume each $\left|S_{\alpha}\right|<\kappa$. By GCH there is a large $\Delta$-system of supports, i.e. there is some $D \subset \kappa^{+},|D|=\kappa^{+}$, and some $S \subset A$ of size $<\kappa$ so that if $\alpha, \beta \in D$ then $S_{\alpha} \cap S_{\beta}=S$. In $M[G]$ the Boolean algebra generated by $J_{\kappa} \cup\left\{x_{\alpha}\right.$ : $\alpha \in D\}$ is still a counterexample to $8(a)$, so we assume without loss of generality that $D=\kappa^{+}$.

We leave $M$ and work in $M_{S}$. Since we are now forcing over $M_{S}$ with $\mathbf{P}_{A-S}, S$ has become irrelevant and we may assume the $S_{\alpha}$ 's are pairwise disjoint. By GCH in $M$ there are at most $\kappa$ many essentially different terms for subsets of $\delta$, so we may assume (again, by possibly shrinking $\left\{\dot{x}_{\alpha}: \alpha<\kappa^{+}\right\}$to a new set of size $\kappa^{+}$) that if $\{\alpha, \beta\},\left\{\alpha^{\prime}, \beta^{\prime}\right\}$ are disjoint subsets of $\kappa$ then there is a permutation $\phi$ of $A-S$ so $\phi\left(\stackrel{\circ}{x}_{\alpha}\right)=\stackrel{\circ}{x}_{\beta}$ and $\phi\left({\stackrel{\circ}{\alpha^{\prime}}}^{\prime}\right)=\stackrel{\circ}{x}_{\beta^{\prime}}$

By the pigeonhole principle, there is some $\gamma$ so that $\left\{\alpha: \exists \beta>\alpha\left(1 \Vdash \dot{x}_{\alpha} \cap \dot{x}_{\beta} \in\right.\right.$ $\left.\left.\stackrel{\circ}{J}_{\gamma}\right)\right\}$ has size $\kappa^{+}$. Pick such a $\gamma$. Since $1 \Vdash\left|\stackrel{\circ}{J}_{\gamma}\right| \leqslant \kappa$ there is some $S^{*} \in M_{S},\left|S^{*}\right|<\kappa$, and $\operatorname{supp}\left(\stackrel{\circ}{\gamma}_{\gamma}\right)=S^{*}$. By throwing out at most $\kappa$ many $S_{\alpha}$ 's we may assume each $S_{\alpha} \cap S^{*}=\phi$. Set $M^{*}=M_{S \cup S^{*}}$ and from now on work in $M^{*}$.

Note that $J_{\gamma} \in M^{*}$. Pick $\alpha, \beta$ so that $1 \Vdash \dot{x}_{\alpha} \cap \dot{x}_{\beta} \in J_{\gamma}$. If $\phi$ permutes $A-(S \cap$ $\left.S^{*}\right)$ then $1 \Vdash \phi\left(\dot{x}_{\alpha}\right) \cap \phi\left(\dot{x}_{\beta}\right) \in J_{\gamma}$. But we already know that if $\{\alpha, \beta\} \cap\left\{\alpha^{\prime}, \beta^{\prime}\right\}$ $=\phi$ then there is some $\phi$ with $\phi\left(\dot{x}_{\alpha}\right)=\dot{x}_{\beta}$ and $\phi\left(\dot{x}_{\alpha^{\prime}}\right)=x_{\beta^{\prime}}$. We've shown that $1 \Vdash \dot{x}_{\alpha} \cap \dot{x}_{\beta} \in J_{\gamma}$ for all $\alpha, \beta<\kappa^{+}$, and we're done.

Proof of 8(b). Again we start in $M[G]$ with a counterexample $X=\bigcup_{\alpha<\rho} J_{\alpha}$, now with $\rho \geqslant \kappa^{+}+1$, each $\left|J_{\alpha}\right|<\kappa$ for $\alpha<\kappa^{+}$, and $\left|J_{1}\right|=\delta<\kappa$. In $M$ there are terms $\dot{x}_{\alpha}$, $\alpha<\kappa^{+}$, so $1 \Vdash \dot{x}_{\alpha} \in A t^{\alpha}(X)$ for all $\alpha<\kappa^{+}$. Again we have a $\Delta$-system of supports and again we may suppose (by moving up to an intermediate model $M_{S}$ ) that in fact the supports are disjoint. Again we define $\gamma_{\alpha, \beta}$ to be the least ordinal for which

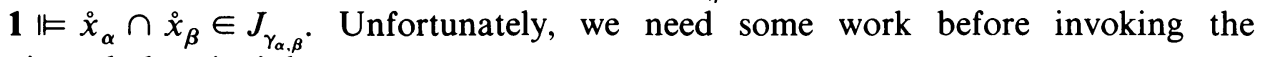
pigeonhole principle. 
Again, each $\operatorname{supp}\left(\dot{J}_{\alpha}\right)$ has size at most $\kappa$. Since the $\operatorname{supp}\left(\stackrel{\circ}{x}_{\alpha}\right)$ 's have size less than $\kappa$, are pairwise disjoint, and there are $\kappa^{+}$many of them, we can find a set $E \in M$, each ordinal in $E$ has cofinality $\operatorname{cf}(\kappa),|E|=\kappa^{+}$, and if $\alpha<\beta \in E$ then $\sup \left(\dot{J}_{\alpha+1}\right) \cap$ $\sup \dot{x}_{\beta}=\varnothing$. Now invoke the pigeonhole principle to find $\alpha \in E$ so that for some $\gamma$ $\left\{\beta \in E: \beta>\alpha\right.$ and $\left.\mathbf{1} \Vdash \dot{x}_{\alpha} \cap \dot{x}_{\beta} \in \dot{J}_{\gamma}\right\}$ is cofinal in $\kappa^{+}$. Let $\gamma$ be the least such, and let $E^{*}=\left\{\beta \in E: \beta>\alpha\right.$ and $\left.\mathbf{1} \Vdash \dot{x}_{\alpha} \cap \dot{x}_{\beta} \in \dot{J}_{\gamma}\right\}$. Note that by $\lambda^{+}-$c.c., $\operatorname{cf}(\gamma)<\kappa$.

Case 1. $\gamma=\alpha+1$. Then for all $\beta \in E^{*} \quad 1 \Vdash \stackrel{\circ}{x}_{\alpha} \cap \stackrel{\circ}{x}_{\beta}=\left(\dot{\circ}_{\alpha} \cup{\stackrel{\circ}{y_{\beta}}}\right)-\stackrel{\circ}{z}_{\beta}$ where $1 \Vdash \dot{y}_{\beta}, \stackrel{\circ}{z}_{\beta} \in{\stackrel{\circ}{\gamma_{\beta}^{\prime}}}^{\prime}$ for some $\gamma_{\beta}^{\prime}<\alpha$. By a counting argument we may shrink $E^{*}$ to a set of size $\kappa^{+}, \bar{E}$, so for some fixed $\gamma^{\prime}$, if $\beta \in \bar{E}-\{\alpha\}$ then $\gamma_{\beta}^{\prime}=\gamma^{\prime}$. By another counting argumment (possibly shrinking $\bar{E}$ again) there are $\stackrel{\circ}{y}, \stackrel{\circ}{z}$ so for all $\beta \in \bar{E}, \stackrel{\circ}{y}=\dot{y}_{\beta}$, $\stackrel{\circ}{z}=\stackrel{\circ}{z}_{\beta}$. Then if $\beta, \xi$ are distinct elements of $\bar{E}$ there is some permutation $\phi$ so

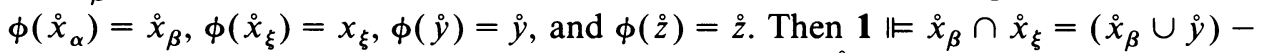
$\dot{z}$. But if we chose $\xi<\beta, \stackrel{\circ}{x}_{\xi}$ is then not an atom of $\dot{x} / \dot{J}_{\xi_{1}}$, since it contains, modulo an earlier ideal, an element of $A t^{\beta}(\stackrel{X}{X})$. And this is a contradiction.

Case 2. $\gamma<\alpha$. Then for $\beta, \delta$ distinct elements of $E^{*}$ there is some permutation $\phi$ so $\phi\left(\dot{x}_{\alpha}\right)=\dot{\circ}_{\beta}, \phi\left(\dot{x}_{\delta}\right)=\dot{\circ}_{\delta}$, and $\phi\left(\dot{\circ}_{\gamma}\right)=\dot{\circ}_{\gamma}$. Hence $1 \Vdash \dot{x}_{\beta} \cap \dot{x}_{\delta} \in \dot{J}_{\gamma}$ for all $\beta$, $\delta \in E^{*}$. But then $M[G] \Vdash\left\{x_{\beta}: \beta \in E^{*}\right\}$ are mutually incompatible mod $J_{\gamma}$, so $M[G] \Vdash$ wd $_{\gamma}(X) \geqslant \kappa^{+}$, which is a contradiction.

All of these negative results are quite peculiar given the following unanswered question: is there a consistent example of a thin-very tall $\mathrm{sBa}$ ? In fact, no consistent examples are known of $\kappa$-thin tall sBa's with fewer than $\kappa$ atoms, for any $\kappa$.

\section{REFERENCES}

1. J. Baumgartner, Almost disjoint sets, the dense set problem, and the partition calculus, Ann. of Math. Logic 10 (1976), 401-439.

2. Iterated forcing, Surveys in Set Theory (A. R. D. Mathias, ed.), London Math. Soc. Lecture Note Ser. vol. 87, Cambridge Univ. Press, 1983, 1-59.

3. G. W. Day, Superatomic Boolean algebras, Pacific J. Math (1967), 479-489.

4. K. J. Devlin, Kurepa's hypothesis and the continuum, Fund. Math. 139 (1975), 23-31.

5. , $\aleph_{1}$-trees, Ann. of Math. Logic 13 (1978), 267-330.

6. I. Juhász and W. Weiss, On thin-tall scattered spaces, Colloq. Math. 90 (1978), 63-68.

7. W. Just, Two consistency results concerning thin-tall Boolean algebras (to appear).

8. R. LaGrange, Concerning the cardinal sequence of a Boolean algebra, Algebra Universalis 7 (1977), 307-312.

9. W. Mitchell, Aronszajn trees and the independence of the transfer property, Ann. of Math. Logic 5 (1972), 21-46.

10. M. Rajagapolan, A chain compact space which is not strongly scattered, Israel J. Math. 23 (1976), $117-125$.

11. S. B. Todorĉević, Some consequences of $M A+\neg w K H$, Topology Appl. 12 (1981), 187-202.

12. M. Wage, Almost disjoint sets and Martin's axiom, J. Symbolic Logic 44 (1979), 313-318.

13. M. Weese, On the classification of compact scattered spaces (Proc. Conf. on Topology and Measure. III), Part 2, Greifswald, 1982, pp. 347-356.

14. On cardinal sequences of Boolean algebras (to appear).

Department of Mathematics, University of Kansas, Lawrence, Kansas 66045 